\title{
SIMULATION OF THE CIRCUITS FOR ELECTRIC VEHICLES CHARGING SYSTEMS
}

\author{
George Onoja IDOKO \\ Politehnica University of Bucharest \\ georgeidoks@gmail.com
}

\begin{abstract}
This paper examines the charging system of electric vehicles and tries to explain the process involved in the operation of an electric vehicle supply equipment and the electric vehicle onboard charging module. The simulation carried out in this paper were done using the power electronics simulation software PSIM. It presents the electric schematic of the proximity pilot connection and the control pilot connection. It also presents the voltage waveforms for both connector pins.
\end{abstract}

\section{INTRODUCTION}

Racing to close the infrastructural gap for charging EVs, many solutions for charging EVs at resident, work and public locations are being considered. Simplification and the entire system and a desire to cut cost, continue to inspire new and more efficient solutions. The variant of Level 2 ac charging appears to increasingly fall in favor of EV owners for its closer to practical charging speeds, cheaper installation and running price tag and great flexibility across a great number of EV models. In this article, the electric vehicle supply equipment (EVSE) is looked at, and the functioning process is simulated using PSIM, an electronic circuit simulation package.

\section{EV AND PLUG-IN HYBRID EV CHARGING}

Essentially, conductive charging, which is using conductive materials to deliver electric energy to the point storage is divided into three functions, two electrical functions and one mechanical function. These three functions must be performed to charge an EV battery from the electric supply network.

Charging of electric vehicles and plug-in hybrids electric vehicles is divided broadly into onboard charging and offboard charging [1] These two charging systems are categorized mainly by the point where the actual charging occurs. Onboard charging happens exclusively onboard the vehicle where the ac power is delivered directly, while offboard charging occurs outside the vehicle and the energy is delivered to the car batteries directly regulated by communication between the car and the charging system. Onboard charging is achieved with the use of an onboard charging module (OBCM). The on-board charging module carries out the function of converting the voltage, power factor correction and supplying the batteries with the needed voltage and current needed to charge them at the proper rate.

\section{ELECTRIC VEHICLE SUPPLY EQUIPMENT}

The electric vehicle supply equipment (EVSE) provides the electric vehicle with the electric energy needed to charge the onboard batteries. More common are level 2 ac electric vehicle supply equipment. These devices provide ac voltage to the vehicle and the onboard charger in the vehicle converts and distribute this power to the battery packs. The EVSE is composed of software to regulate charge rate and state, conductors to transfer the power and safety parameters to ensure safe delivery and management of power through its circuits [2]. According to the SEA an EVSE comprises of the conductors, ungrounded, grounded, and 
electric vehicle connectors, attachment plugs, power outlets and all other accessories, or devices installed precisely to deliver energy to the electric vehicle [3].

\section{CHARGING LEVELS}

The J1772 is a charging standard proposed by the Society of Automotive Engineers, abbreviated to SAE. The SAE International is an organization with the mission of advancing mobility knowledge and presenting solutions for the benefit of humanity [4]. The J1772 plug is a 5-pin plug capable of delivering single-phase ac power up to $240 \mathrm{~V}$ ac at up to 80 amperes. The 5-pin plug designed by Yazaki supports a wide range of ac power levels from $1.44 \mathrm{~kW}$ to $19.2 \mathrm{~kW}$. Additionally, the $\mathrm{J} 1772$ standard has a variant with 7-pins, this version referred to as combined charging standard (CCS) can provide in addition to ac charging, dc fast charging.

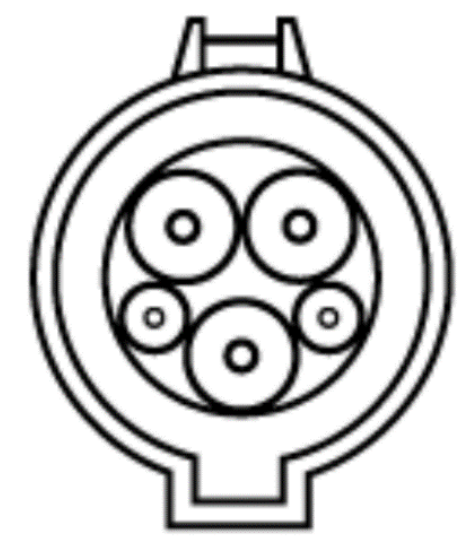

Figure 1. The J1772 standard plug was designed by Yazaki [5].

Under the SEA (Society of Automotive Engineers) there are four conductive coupling charging standards. These charging standards apply to conductive charging in surface vehicles.

Table 1. The charging levels of Electric vehicles are recognized under the J1772 standard.

\begin{tabular}{l|l}
\hline Ac level 1 & up to $1.92 \mathrm{~kW}$ \\
Ac level 2 & up to $19,2 \mathrm{~kW}$ \\
Dc level 1 & up to $48 \mathrm{~kW}$ \\
Dc level 2 & up to $400 \mathrm{~kW}$
\end{tabular}

All EV's and PHEVs have two different dc batteries, a $12 \mathrm{~V}$ battery to power the low voltage electronics of the EV like the wipers, electric windows, lights etc. and a high voltage battery or traction battery. The high voltage battery is usually at around $300 \mathrm{~V}$ dc to $800 \mathrm{~V} \mathrm{dc}$ and supplies power to the inverter that drives the electric traction motor(s). It also supplies power to the dc to ac converter in the case of a vehicle to grid power flow and dc to dc converter used to charge the low voltage battery.

\section{CHARGING MODES} the EV.

Charging modes simply refer to the physical components involved in the charging of 
Charging Mode 1: This charging mode offers slow charging from a regular ( $230 \mathrm{~V}$ in the EU of $170 \mathrm{~V}$ in America) electrical socket (single- or three-phase)

Charging Mode 2: This also offers slow charging from a regular socket but with some EV specific protection arrangements to limit the current and automatic turn off the switch.

Charging Mode 3: Mode 3 can slow or fast charge the EV using a specific EV multi-pin socket with control and protection functions, mode 3 charging is often implemented in level 3 charging.

Charging Mode 4: Fast charging using some special charger technology such as CHAdeMO, the tesla charger etc. this charging mode often delivers direct current to the EV [6,7].

\section{DETAILS OF THE CONNECTOR PLUG}

In this article the SAE J1772 plug is looked at, other charging plugs include CCS combo 1 type 1 , CSS combo 2 type 2 and CHAdeMO Yazaki.

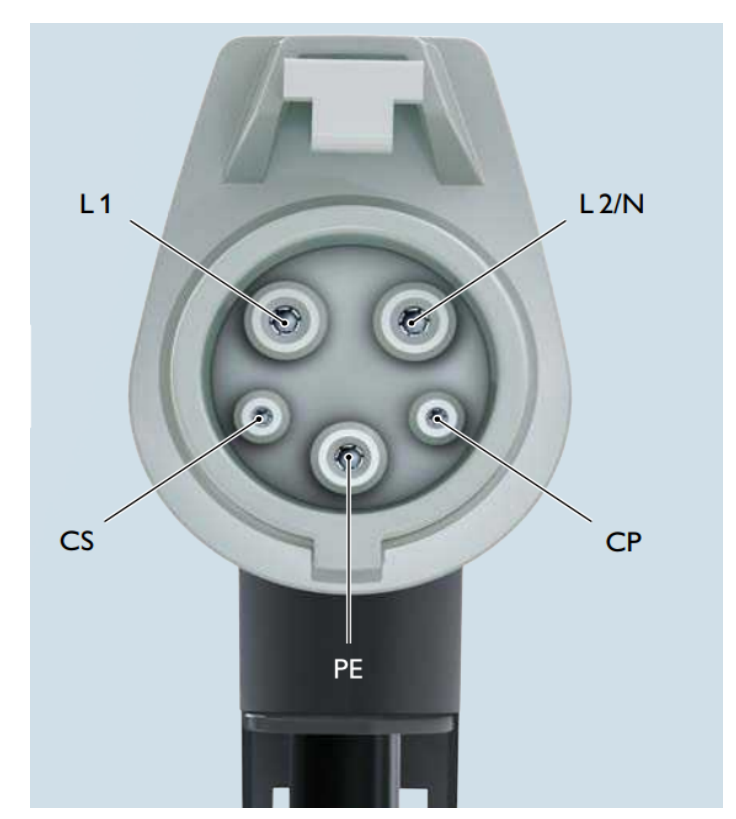

Figure 2. XY plane image of an SAE J1772 [8].

L1 - Mains 1: This pin will carry the alternating current that will go into the onboard charging module.

L2/N - Mains 2 / Neutral

$\mathrm{PE}$ - protective Earth

CP - Control Pilot: the EV would communicate with the EVSE via this pin, information like the EV being ready to receive a charge and the amount of energy needed is transferred through this pin.

CS - Connection Switch, proximity detection or proximity pilot: the EVSE detects that an EV is connected to it via this pin. When the EV detects a voltage on this pin the car is forbidden from moving.

The difference between this plug and its 3 phased counterparts is the presence of an additional 2 pin prong to handle the extra phases. The figure below shows the electronic schematic of an EV plugged into an EVSE. 


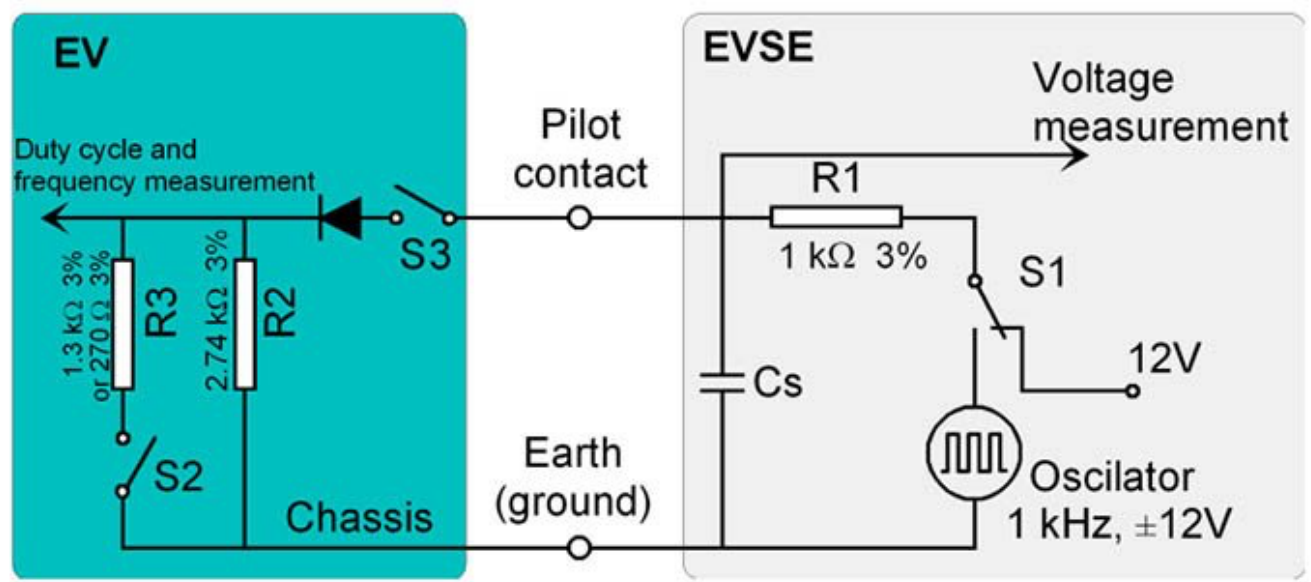

Figure 3. Electric schematic of EVSE connection to EV [9].

The proximity pilot has a $12 \mathrm{~V}$ DC across it that drop when the charge plug is connected to the EV. The voltage on the proximity pin is measured against the ground pin. When the controller on the EVSE detects the voltage drop, the S1 switch as shown in figure 2 above is closed and the control pilot sends a square wave signal to the EV. The signal on the control pilot measures $9 \mathrm{~V} \mathrm{dc}$ when there is a connection between the EV and the EVSE, but the EV is not ready to charge. When the EV is ready to charge the S2 switch is closed and the voltage on the control pilot drops to $6 \mathrm{~V}$.

\section{PSIM SIMULATION}

The simulation is carried out using the software PSIM. It attempts to simulate the functioning processes of an EVSE at ac level 2. Figure 3 shows a model of the proximity pilot of the J1772 charger. The relay is used to simulate the charging plug connection to the vehicle. Figure 6 shows a model of the control pilot connection with the EV. Timed relay switches are used to simulate the moment of connection and engage the resistors to mimic the processes during charging.

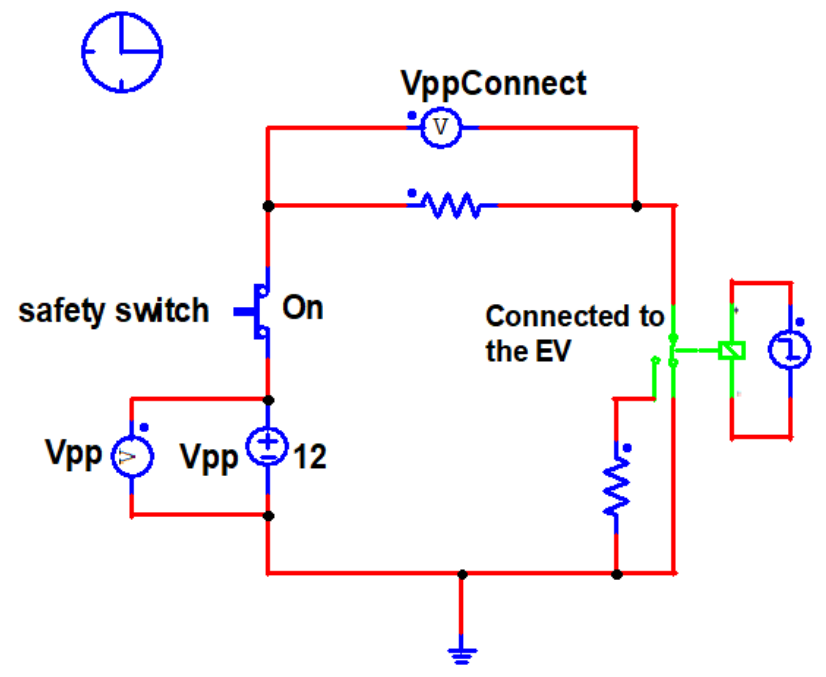

Figure 4. Schematic of Proximity pilot connection. 


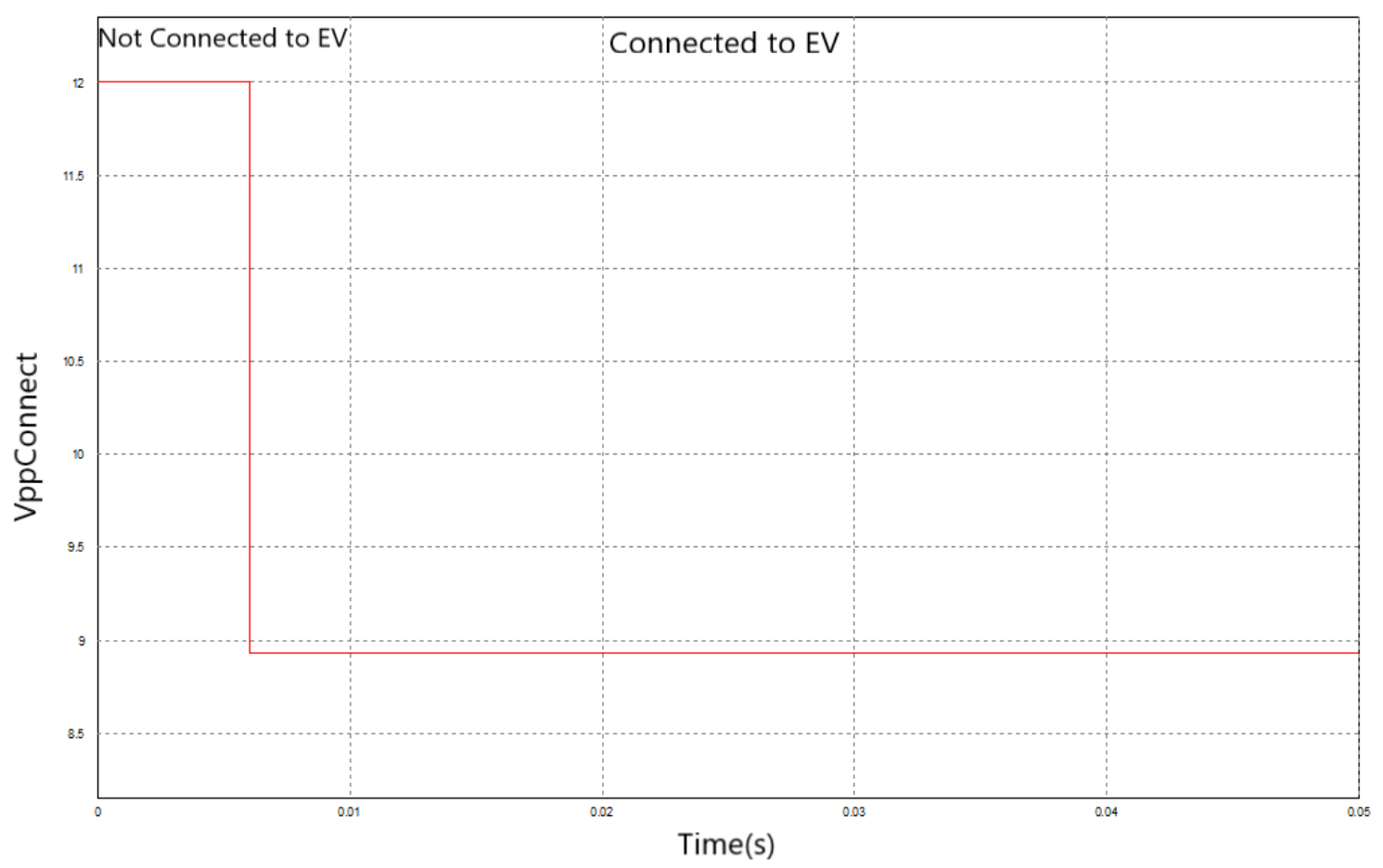

Figure 5. Voltage waveform on the proximity pilot pin.

The proximity pilot alerts the vehicle that there is a connection between the EVSE and the electric vehicle. The voltage drop at the proximity pin occurs once the plug is connected to the vehicle.

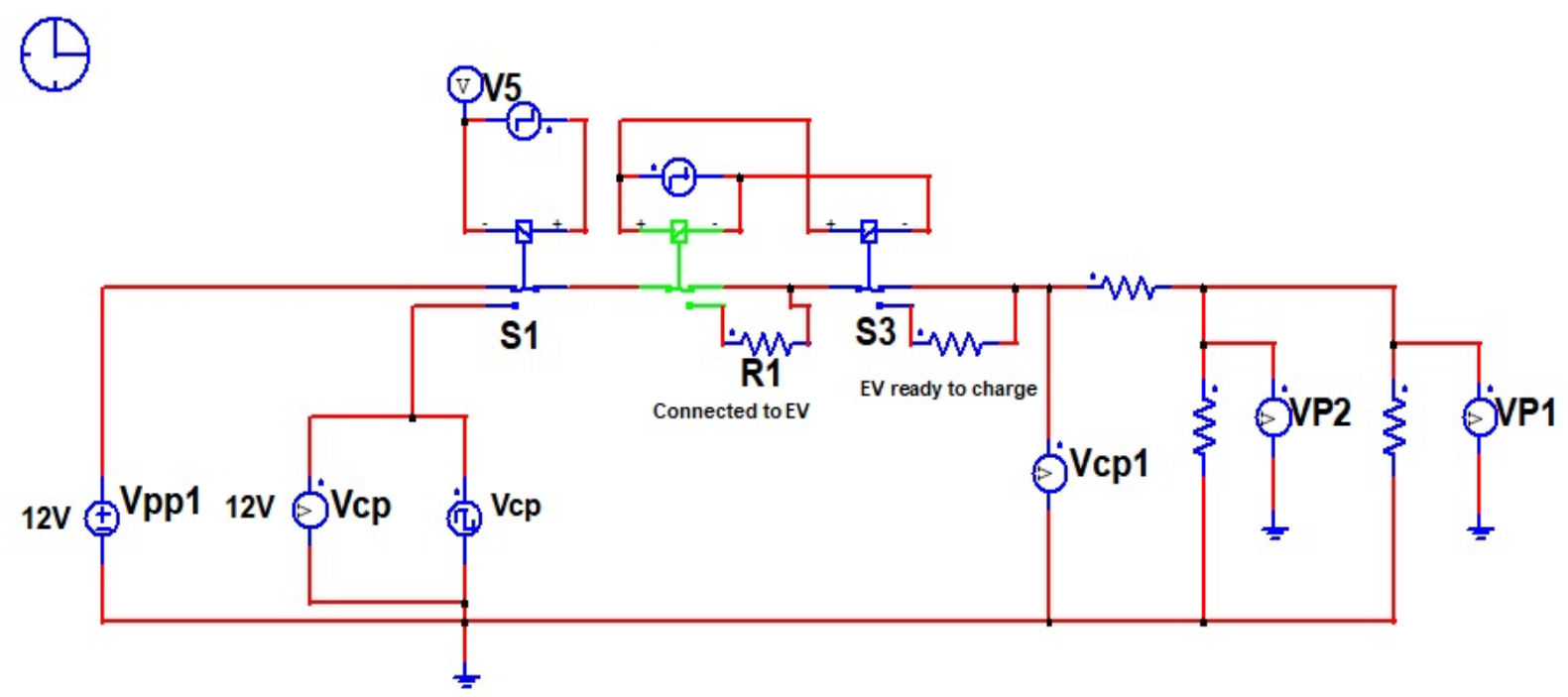

Figure 6. Schematic of the Control pilot pin connection. 


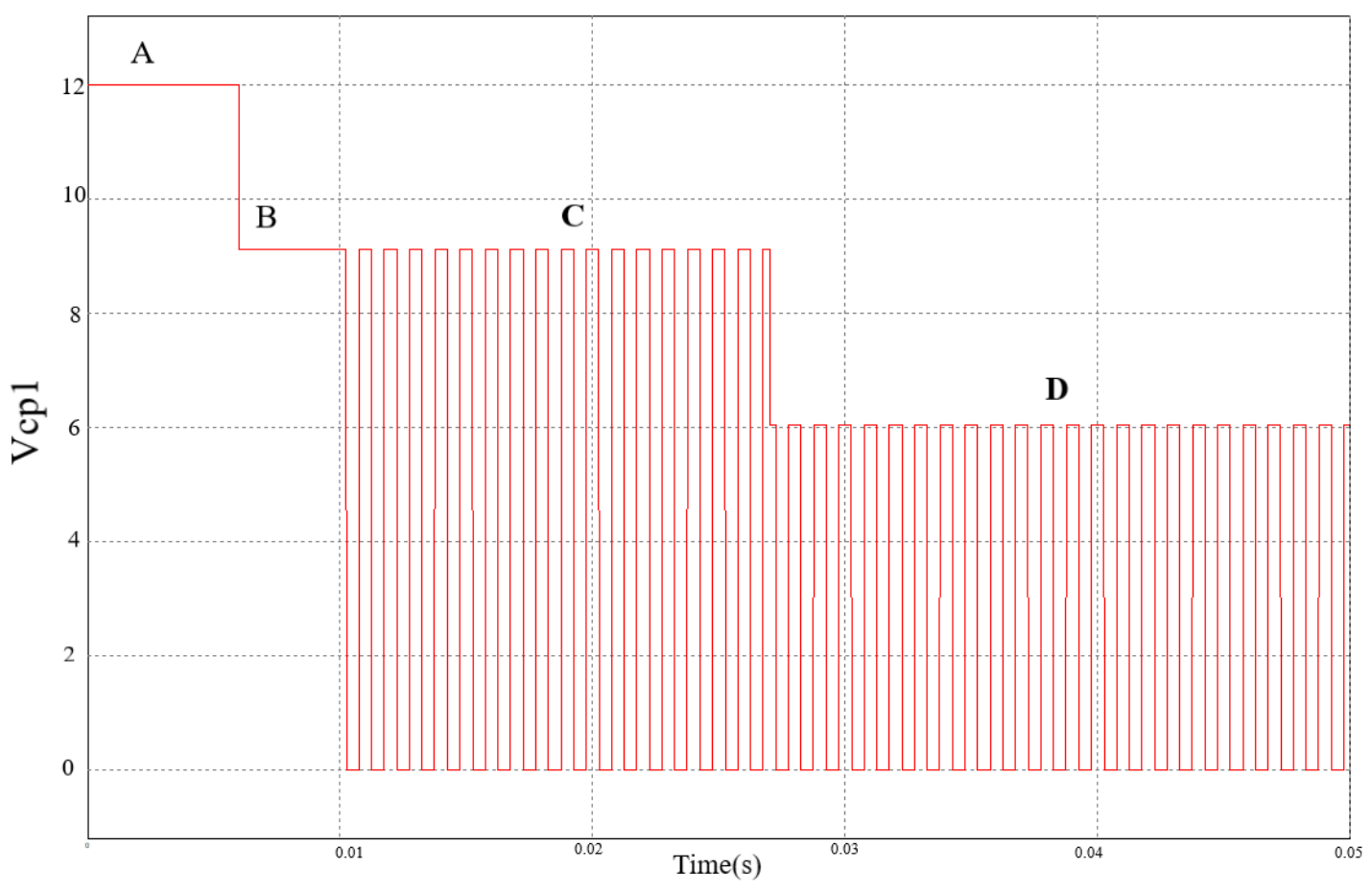

Figure 7. Voltage waveform on the Control pilot.

The voltage waveform from the above diagram shows the various steps of the voltage measurement at the control pilot before charging occurs. At step A the voltage at the pin measures $12 \mathrm{~V}$ as it is not connected to the vehicle now. There is a voltage drop at step B, this happens because the plug has now been connected to the vehicle. At step C, the control pilot begins to send a $1 \mathrm{kHz}$ PWM signal with an amplitude of $9 \mathrm{~V}$ to the vehicle, signaling that the EVSE is ready to begin charging. Step $\mathrm{D}$, the vehicle is ready to receive the charge and closes its contactor to complete the circuit at the power line. The voltage on the control pilot then drops to $6 \mathrm{~V}$.

\section{CONCLUSIONS}

The electric vehicle service equipment (EVSE) facilitated the delivery of power safely from the grid to the electric vehicle. The principal functions of an EVSE are to regulate the electric current flowing into the EV, assuring that it is safely under the maximum current the electric vehicle can handle, the EVSE automatically disconnect when a hardware fault is detected and to prevent current from flowing when there is no connection between the EV and the EVSE [10]. In the case of commercial EVSEs, other functionalities like energy metering, user data identification, payment processing and IoT functionalities may also be included.

The functioning of an EVSE is simulated using PSIM. The electronic schematics were built to mimic the plugging and communication process between the EVSE and the EV. Level 2 ac charging appears to continuously increase in popularity as it does not need any new infrastructure to be set up. They can function on the available $230 \mathrm{~V}$ ac, and the charging and power conversion is handled by the onboard charging module present in the EV. The adaptation of the combined charging system attempts to combine a solution for fast offboard dc charging and onboard ac charging into one [11]. As the electronics in the OBCM get better and more efficient, onboard charging would get faster as they would be able to handle greater power. 


\section{REFERENCES}

[1] R. Rebeka, G. Velimir, P. Denis and T. Danijel, "Overview of charging modes and connectors for the electric vehicles," IEEE, 2019.

[2] A. Moldoveanu, "NEMA.org,"NEMA; Electric Vehicle Supply Equipment/System, [Online]. Available: https://www.nema.org/directory/products/view/electric-vehicle-supply-equipmentsystem. [Accessed 4 November 2021].

[3] SAE EV Charging Systems Committee, "SAE Electric Vehicle Conductive Charge Coupler," SAE, California, 2001.

[4] SAE International , "About SAE International," SAE International, [Online]. Available: https://www.sae.org/about. [Accessed 281 2022].

[5] chargeHub, "2020 Guide on how to charge your Electric Car with charging stations.," Electric Vehicle Charging Guide | ChargeHub, [Online]. Available: https://chargehub.com/en/electriccar-charging-guide.html. [Accessed 271 2022].

[6] P. Vedran Bobanac and P. Assoc. prof. Hrvoje, "Energy Storage, Electric Vehicles as Energy Storage Providers," [Online].

[7] B. Ali, "EV Charging Definitions, Modes, Levels, Communication Protocols and Applied Standards," ResearchGate, p. 42, January 2020.

[8] PHOENIX CONTACT, "CHARGING TECHNOLOGY FOR E-MOBILITY," 2017. [Online]. Available: https://www.phoenixcontact.com/en-gb/products. [Accessed 261 2022].

[9] R. Mihai, G. Adrian, R. Felseghi Raluca Andreea and F. Afanasov, "The ElectricalVehicle Simulator for Charging Station in Mode 3 of IEC 61851-1 Standard," Research Gate, vol. 13, p. 176, December 2019.

[10] TEXAS INSTRUMENTS, "SAE J1772-Compliant Electric Vehicle Service Equipment," TIDUER6, TEXAS INSTRUMENTS, 2019.

[11] K. Rajashekara, "Present Status and Future Trends in Electric Vehicle Propulsion Technologies," IEEE journal of emerging and selected topics in power electronics, vol. 1, p. 8, 2013.

[12] E. C. P. Company, "Electric Car Parts Company," Electric Car Parts Company, [Online]. Available: https://www.electriccarpartscompany.com/J1772-Plugs-with-Cable--USA-Stock-brLevel-1-br-15A-16A-br-120V-240V_p_381.html. [Accessed 411 2021]. 